\title{
Avulsão traumática do nervo óptico por projétil de arma de fogo de grosso calibre: relato de caso
}

\author{
Traumatic optic nerve a vulsion by high caliber bullet:case report
}

\author{
Rogério de Almeida Tárcia ${ }^{1}$
}

Trabalho realizado no serviço de oftalmologia do Hospital de Pronto-Socorro João XXIII Fundação Hospitalar do Estado de Minas Gerais - FHEMIG - Belo Horizonte (MG) - Brasil.

${ }^{1}$ Médico oftalmologista do Hospital de Pronto-Socorro João XXIII Fundação Hospitalar do Estado de Minas Gerais - FHEMIG - Belo Horizonte (MG) - Brasil. Assistente do Departamento de Ecografia Ocular do Hospital São Geraldo - Hospital das Clínicas da Universidade Federal de Minas Gerais - UFMG - Belo Horizonte (MG) - Brasil.

Endereço para correspondência: Rogério de Almeida Tárcia. Av. João Pinheiro, 146 - 13ํandar - Belo Horizonte (MG) CEP 30130-922

E-mail: almeidatarcia@yahoo.com.br

Recebido para publicação em 04.04.2005

Aprovação em 07.12.2005

\begin{tabular}{|l|}
\hline RESUMO \\
\hline A avulsão traumática do nervo óptico ocorre mais comumente devido a \\
traumas contusos óculo-orbitários. A avulsão do mesmo por projétil de \\
arma de fogo de grosso calibre é causa excepcional desta lesão e deve ser \\
pensada no manejo dos pacientes vítimas de lesões por arma de fogo na \\
região cefálica. O presente trabalho descreve o primeiro caso documenta- \\
do desta singular lesão ao nervo óptico e tece comentários sobre as \\
diversas outras causas relatadas, mecanismos fisiopatológicos, histologia \\
e condutas.
\end{tabular}

Descritores: Traumatismos oculares; Ferimentos por arma de fogo; Traumatismos do nervo óptico; Enucleação ocular; Relatos de casos [Tipo de publicação]

\section{INTRODUÇÃO}

Estima-se, pelo menos, 30.000 mortes em 300.000 casos de lesões gerais por armas de fogo nos Estados Unidos, todos os anos ${ }^{(1)}$. Dentro deste contexto, as lesões oculares e orbitárias por projéteis de arma de fogo de grosso calibre são fortes contribuintes para a morbidade em grandes pronto-socorros, em todo o mundo.

O espectro destas lesões é muito amplo, podendo variar desde simples contusão do globo ocular, até traumas oculares abertos de grandes proporções. Associam-se comumente a lesões crânio-encefálicas e a fraturas do terço médio da face. Quando exclusivas da órbita óssea e de seu conteúdo, são consideradas raras $^{(2)}$ e, quando exclusivas do bulbo ocular são excepcionais ${ }^{(3)}$.

Diversos agentes etiopatogênicos são relatados na literatura como causadores de avulsão traumática do nervo óptico, junto à sua inserção no globo ocular. Dentre eles, com maior freqüência, encontramos os traumas contusos óculo-orbitários, por diversas causas: queda ${ }^{(4-5)}$; nas práticas esportivas, em especial no basquetebol ${ }^{(6)}$, devido aos dedos do adversário que adentram a região orbitária; por explosão doméstica ${ }^{(7)}$; por acidentes automobilísti$\cos ^{(5,8-11)}$; por coice de animais ${ }^{(6)}$. Há relatos de enucleação por arrancamento (agressão) ${ }^{(12)}$, e por auto-enucleação, também chamado de edipismo, encontrados em doentes psicóticos ou em viciados em drogas ${ }^{(13)}$.

Este trabalho tem por objetivo relatar um caso de avulsão traumática do nervo óptico, junto à sua inserção no globo ocular, por projétil de arma de fogo de grosso calibre. As peculiaridades do caso são discutidas, já que não há relato na literatura médica, de arrancamento da cabeça do nervo óptico, por este tipo de agente agressor, com a preservação completa do remanescente do bulbo ocular. 


\section{RELATO DE CASO}

Paciente G.G., 38 anos, casado, masculino, faioderma, operador de máquinas, procedente de Contagem, região metropolitana de Belo Horizonte (MG), deu entrada na emergência do Hospital de Pronto-Socorro João XXIII, na noite do dia 14/01/05, vítima de agressão por arma de fogo na região cefálica.

Inicialmente avaliado por um neurocirurgião, encontravase lúcido, cooperativo, sem déficit motor, com índice de Glasgow 15. Mantinha-se estável do ponto de vista respiratório e hemodinâmico, quando fora solicitado o exame de tomografia computadorizada (TC).

Constatou-se dois trajetos de projétil de arma de fogo de grosso calibre, ambos extra-cranianos (Figura 1). O primeiro entrou pelo nariz e se alojou na articulação têmporo-mandibular direita, causando uma paralisia facial periférica ipsilateral.

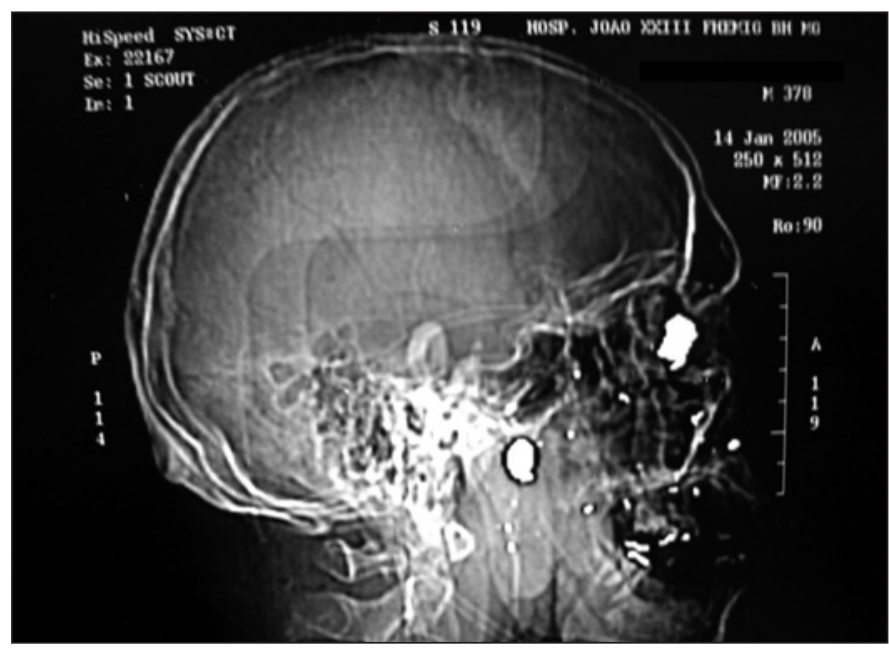

Figura 1 - Topograma da região da cabeça, onde se observam os dois projéteis de arma de fogo de grosso calibre, um na região da articulação têmporo-mandibular direita e o outro na região orbitária posterior direita

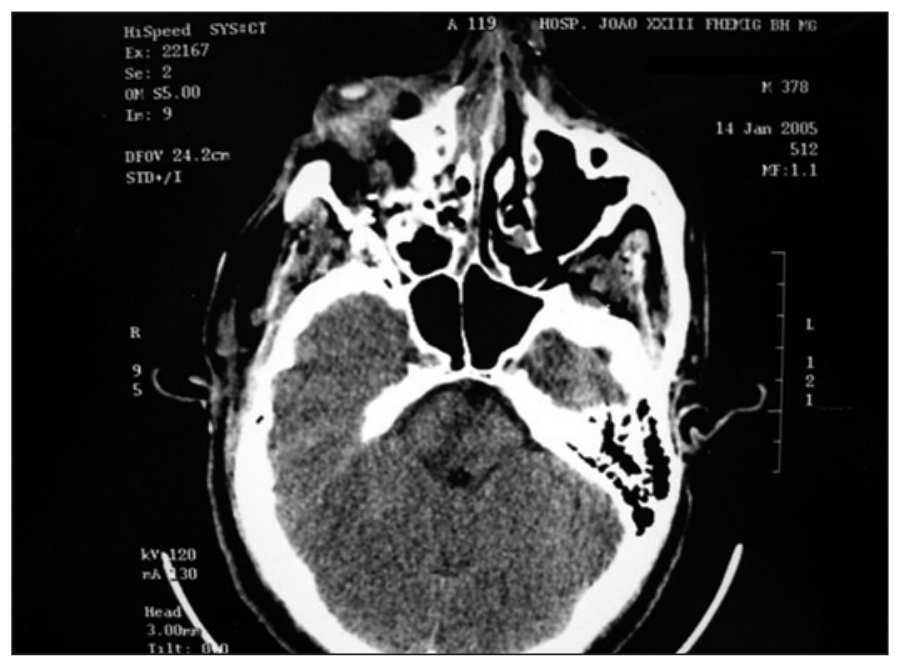

Figura 2 - Corte axial de tomografia computadorizada, onde se nota na órbita direita solução de continuidade do nervo óptico, próximo ao globo ocular, que se mantém esférico
O segundo projétil entrou pela nuca, margeando a coluna vertebral, sem no entanto atingí-la; fraturou o osso maxilar direito e assoalho orbitário, alojando-se na região intra-orbitária posterior direita, dentro do cone muscular.

Já na TC notava-se a presença do projétil na órbita direita, hemorragia retrobulbar de médio volume e uma solução de continuidade do nervo óptico, junto ao globo ocular, sugerindo avulsão traumática do mesmo (Figura 2). O bulbo ocular, por sua vez, mantinha sua esfericidade, com bolsões hemorrágicos coroidianos e musculatura extra-ocular íntegra, inserida normalmente.

Foram solicitadas avaliações da cirurgia buco-maxilo-facial (tratamento inicial conservador) e da oftalmologia.

Ao exame oftalmológico, o olho direito encontrava-se sem percepção luminosa, com midríase paralítica e com discreta opalescência corneana, devido ao ressecamento por exposição. Havia protrusão extrema do globo ocular, deslocado inferiormente, com grande hemorragia subconjuntival e quemose.

Devido à marcante presença do projétil retrobulbar e à impossibilidade de recuperação funcional do olho, o paciente foi operado sob anestesia geral, para se completar a enucleação (Figura 3), com ligadura e ressecção dos músculos retos, remoção do projétil e sutura da cavidade em três planos: a) plano profundo (chuleio interrompido) com Vicryl ${ }^{\circledR} 5-0$; b) cápsula de Tenon com pontos separados de Vicryl ${ }^{\circledR} 5-0$, e c) plano conjuntival com pontos separados de Vicryl ${ }^{\circledR}$ 6-0. Não foram colocados implantes orbitários.

A peça anatômica (Figura 4) mostrava o defeito circular completo na esclera posterior, no lugar onde o nervo óptico e sua bainha estariam inseridos. Um tecido negro (aparentemente úvea ou sangue) era visível através deste buraco. O exame anátomopatológico encontra-se detalhado na legenda da figura 5.

O projétil removido era de calibre 38 , feito de chumbo e foi registrado na Delegacia Especializada em Crimes Contra a Vida, órgão da Policia Civil do Estado de Minas Gerais, para posterior anexação ao inquérito criminal (Figura 6).

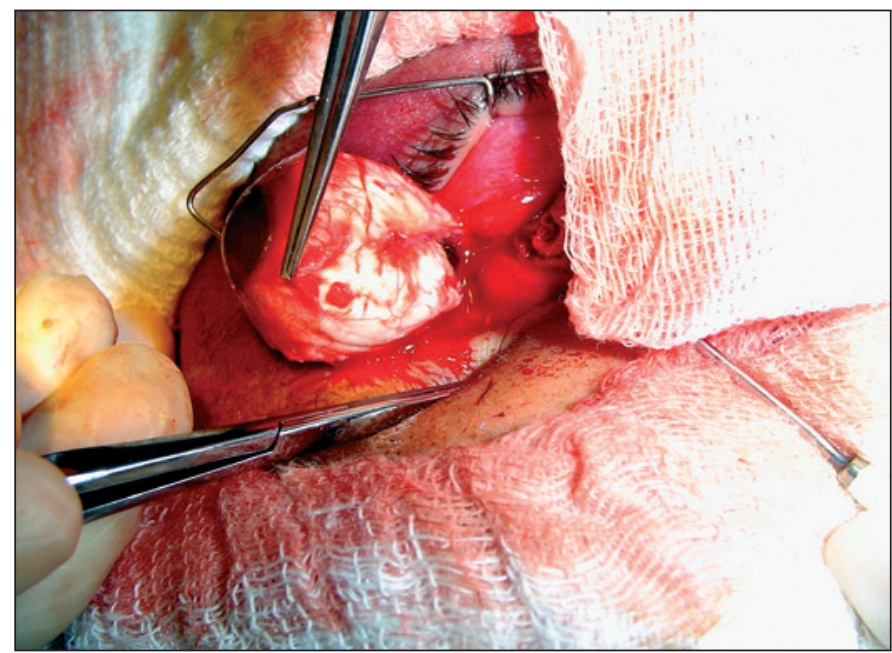

Figura 3 - Aspecto per-operatório de enucleação primária do globo ocular, vendo-se o defeito circular posterior, onde estaria inserido o nervo óptico 


\section{DISCUSS ÃO}

O termo avulsão vem do verbo latino vellere: "arrancar, apartar violentamente". A preposição latina $a b$ : "afastamento, separação de um ponto no espaço ou no tempo", reforça o sentido semântico da palavra. Diante das letras labiais sonoras $\mathbf{m}, \mathbf{v}$ e $\mathbf{b}$, a preposição $a b$ se reduz a $a$. Alguns autores

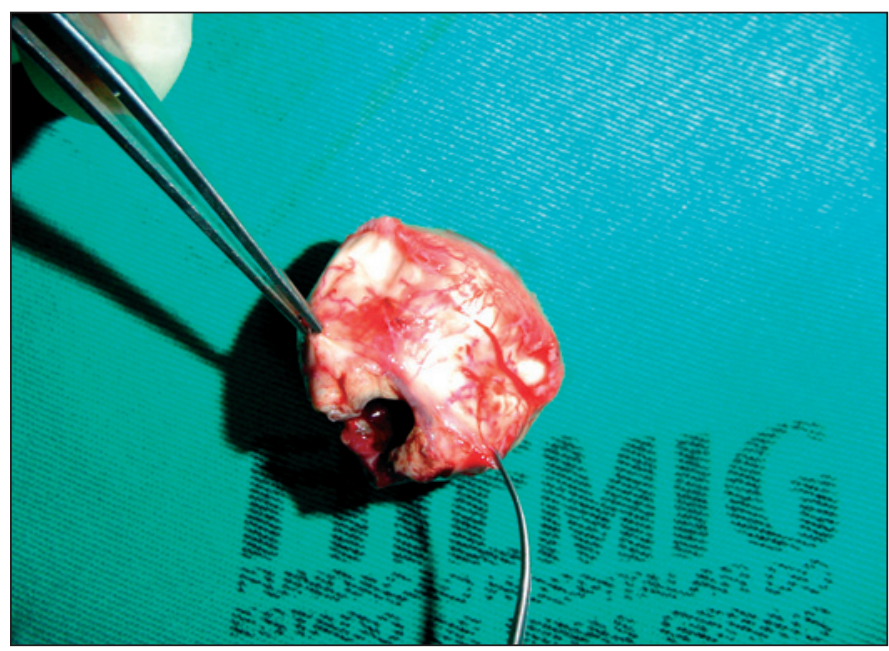

Figura 4 - Peça anatômica (globo ocular direito) com defeito circular posterior, donde se vê um tecido negro, aparentemente úvea ou sangue

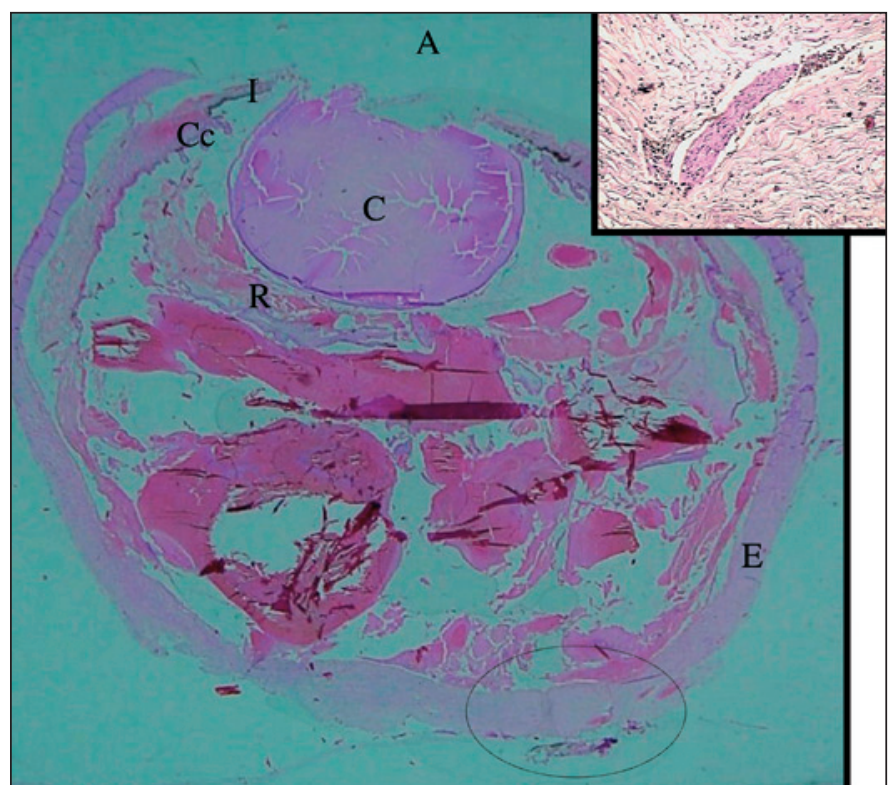

Figura 5 - Fotografia de lâmina histológica de globo ocular, corada por Hematoxilina-Eosina (HE), mostrando desorganização acentuada e difusa da arquitetura das estruturas intra-oculares, com descolamento total de retina, hemorragia, exsudato fibrino-leucocitário e avulsão do nervo óptico (ponto de inserção demarcado com pontilhado). No detalhe (canto superior direito), corte histológico de esclera (HE, 40X) com resquício do nervo avulsionado em meio às fibras colágenas, apresentando hemorragias punctuais e leucócitos esparsos na periferia. (A: anterior; I: íris; E: esclera; Cc: corpo ciliar; R: retina; C: cristalino). Patologista responsável: Dr. Paulo Guilherme de Oliveira Salles. Laboratório Dairton Miranda de Belo Horizonte, MG) preferem o termo evulsão, também derivado de vellere, mas com o prefixo ' $e$ ', que tem o sentido de "para fora"(5,7,9,14). Consideram que quando o globo ocular é deslocado "para fora" da sua posição anatômica normal, este termo seria o mais adequado. No entanto, as palavras são sinônimas, não cabendo este tipo de diferenciação.

A lesão do nervo óptico na sua inserção junto ao globo ocular é facilitada pela vulnerabilidade das fibras nervosas ao nível do disco óptico e pela fragilidade da parede escleral, aqui representada apenas pela lâmina crivosa. A lâmina crivosa é formada pela extensão de feixes colágenos e fibras elásticas dos $2 / 3$ internos da esclera, sem resistência tecidual ${ }^{(4)}$.

Considerando esta fragilidade anatômica natural, diversos mecanismos fisiopatológicos foram postulados na tentativa de explicar como o nervo óptico é avulsionado, na dependência, obviamente, da magnitude e da direção das forças externas envolvidas ${ }^{(5)}$.

Em primeiro lugar, temos as forças rotacionais sobre o globo, que não são acompanhadas pelo nervo óptico, que se rompe. Em segundo, temos as lesões diretas sobre o globo ocular, criando uma força 'explosiva' interna, que é capaz de romper a lâmina crivosa no sentido posterior, mas não é capaz de causar uma ruptura do bulbo, que se mantém formado. O terceiro mecanismo, provocado por lesões indiretas, ocorre quando uma força propulsora súbita desloca o globo anteriormente, devido ao aumento exagerado da pressão intra-orbitária retro-bulbar ${ }^{(4-5,14)}$.

No caso em questão, esta força propulsora foi gerada pelo projétil de arma de fogo que, ao adentrar a região orbitária posterior, projetou o globo ocular para frente, rompendo, desta forma, as fibras nervosas do nervo óptico, junto à sua inserção posterior (Figuras 1 a 6).

Quando a avulsão traumática do nervo óptico ocorre por forças indiretas causadoras de luxação do globo (enucleação

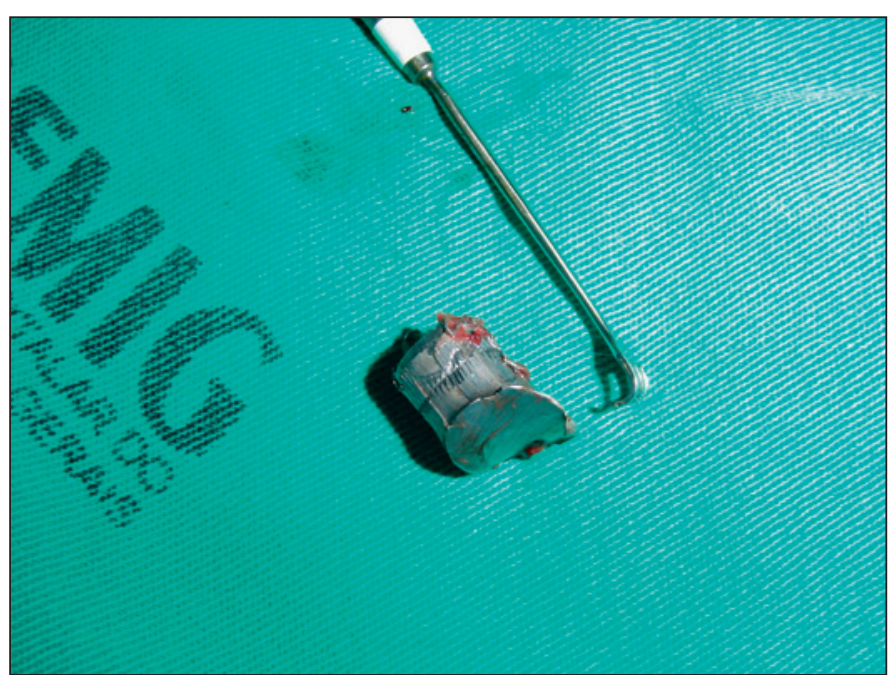

Figura 6 - Projétil de arma de fogo, calibre 38, removido da região orbitária posterior direita do paciente 
traumática), a conduta cirúrgica deve ser tomada imediatamente, assim que possíveis lesões intra-cranianas forem excluídas.

A exploração orbitária deve ser feita em busca de corpos estranhos, sinais de rupturas do globo e para evacuação de hemorragias. Qualquer tipo de enucleação traumática pode evoluir para infecção orbital, fístula liquórica, hemorragia subaracnóidea, meningite e infartos hipotalâmicos, com disfunções endócrinas ${ }^{(12)}$.

Alguns autores preconizam o rápido reposicionamento do globo de volta para a órbita, quando não há rupturas posteriores no globo ocular (avulsão do nervo óptico apenas nas suas porções pré-laminar e laminar crivosa). Nestes casos, a diminuição do edema e a melhora do fluxo sangüíneo preveniria a isquemia e o desenvolvimento de phthisis bulbi ${ }^{(11)}$.

Outros autores concordam que o tratamento principal das luxações do globo ocular com avulsão do nervo óptico é a enucleação primária. No entanto, relatam um caso de uma criança de 18 meses, na qual o globo luxado fora reposicionado para que houvesse um crescimento facial simétrico. A simetria facial foi garantida em todo o período de acompanhamento de 5 anos $^{(15)}$.

Kiratli et al. (1999) preferiram restaurar a musculatura extraocular lesada e, mesmo na presença de uma avulsão do nervo óptico, reduziram a luxação do globo, em grave trauma órbitofacial. Consideram que há um ganho psicológico inicial importante e que, em caso de atrofia ocular, a prótese pode ser adaptada sobre o próprio olho do doente, com uma mobilidade superior ${ }^{(16)}$.

A enucleação primária no trauma ocular deve ser realizada toda vez em que ocorrer perda tecidual grande, impossibilitando tanto a restauração funcional e anatômica do globo, quanto a evisceração com implante orbitário. Desta forma, não haverá contato do pigmento uveal com o sistema imune, considerado o grande responsável pela agressão imunológica contra o olho contralateral, afecção esta chamada de oftalmia simpática ${ }^{(2)}$. Apesar de sua raridade, ocorrendo no máximo em $3-5 \%$ dos casos de traumas penetrantes graves, a oftalmia simpática deve ser considerada uma ameaça nestes casos ${ }^{(1-2)}$.

A avulsão traumática da cabeça do nervo óptico por projétil de arma de fogo de grosso calibre é uma causa incomum desta lesão e deve ser pensada no manejo destes pacientes. A conduta a ser tomada dependerá de cada caso, sendo a enucleação primária a escolhida, principalmente nos casos em que há luxação do globo, para fora dos limites orbitários anteriores.

\section{ABSTR ACT}

Traumatic optic nerve avulsion occurs most commonly after blunt ocular trauma. Optic nerve avulsion by high caliber bullet is exceptional and must be thought of in the management of those patients victims of gunshot wounds to the globe and orbit. The present paper reports the first documented case of this singular condition of optic nerve injury and comments on several other related causes, pathophysiology mechanisms, histology and management.

Keywords: Eye injuries; Wounds, gunshot; Optic nerve injuries; Eye enucleation; Case reports [Publication type]

\section{REFERÊNCIAS}

1. Chu A, Levine MR. Gunshot wounds of the eye and orbit. Ophthalmic Surg. 1989;20(10):729-36.

2. Grant WF Jr, Swan KG. Gunshot wounds of the orbit. J Trauma. 1980;20(9): 809-11.

3. Tárcia RA. Trauma ocular por projétil de arma de fogo de grosso calibre sem envolvimento ósseo orbitário. Rev Bras Oftalmol. 2003;62(12):889-93.

4. Lima SC, Mattos Filho H, Flores IB, Alvim MM. Avulsão do nervo óptico. Rev Bras Oftalmol. 1988;47(2):111-4

5. Morris WR, Osborn FD, Fleming JC. Traumatic evulsion of the globe. Ophthal Plast Reconstr Surg. 2002;18(4):261-7. Comment in: Ophthal Plast Reconstr Surg. 2003;19(3):253-4; author reply 254

6. Foster BS, March GA, Lucarelli MJ, Samiy N, Lessell S. Optic nerve avulsion. Arch Ophthalmol. 1997;115(5):623-30. Erratum in: Arch Ophthalmol. 1997;115(8):1070.

7. Kovacs M. [A case of evulsion of the anterior optic nerve] Klin Monatsb Augenheilkd. 1971;158(6):833-6. German.

8. Espaillat A, To K. Optic nerve avulsion. Arch Ophthalmol. 1998;116(4):540-1.

9. Pillai S, Mahmood MA, Limaye SR. Complete evulsion of the globe and optic nerve. Br J Ophthalmol. 1987;71(1):69-72.

10. Tsopelas NV, Arvanitis PG. Avulsion of the optic nerve head after orbital trauma. Arch Ophthalmol. 1998;116(3):394.

11. Bajaj MS, Pushker N, Nainiwal SK, Balasubramanya R. Traumatic luxation of the globe with optic nerve avulsion. Clin Experiment Ophthalmol. 2003;31(4): 362-3.

12. Shneck M, Oshry T, Marcus M, Lifshitz T. Attempted bilateral manual enucleation (gouging) during a physical assault. Ophthalmology. 2003;110(3): 575-7.

13. Krauss HR, Yee RD, Foos RY. Autoenucleation. Surv Ophthalmol. 1984;29 (3):179-87.

14. Sanborn GE, Gonder JR, Goldberg RE, Benson WE, Kessler S. Evulsion of the optic nerve: a clinicopathological study. Can J Ophthalmol. 1984;19(1):10-6.

15. Vogt P, Motsch S, Muhlendyck H, Hommerich CP. [Management of unilateral traumatic bulb luxation with disruption of the optic nerve] HNO. 2003;51(2): 146-50. German.

16. Kiratli H, Tumer B, Bilgic S. Management of traumatic luxation of the globe. A case report. Acta Ophthalmol Scand. 1999;77(3):340-2.

Nos artigios enviados para publicação, o nome dos autores e suas afiliações devem estar completos. Isso facilitará a indexação e os links com as bases de dados e o CV Lates. 